\title{
Delay of Geo/Geo/1 N-limited Nonstop Forwarding Queue
}

\author{
Yutae Lee \\ Department of Information and Communications Engineering, Dongeui University
}

\begin{abstract}
-
Nonstop forwarding is designed to minimize packet loss when a management system fails to function. A system with non-stop forwarding can continue to forward some packets even in the event of a processor failure. We consider a Geo/Geo/1 N-limited nonstop forwarding queue. In the queueing system, when the server breaks down, up to customers can be serviced during the repair time.The delay distribution of customers is given by matrix geometric analysis.
\end{abstract}

Keywords-Delay, Geo/Geo/l queue, N-limited, nonstop forwarding

Date of Submission: 09 February 2017 Date of Accepted: 25 February 2017

\section{INTRODUCTION}

Queueing systems with servers subject to breakdowns and repairs have been studied extensively [1, 2]. Recently, Falin [3], Lee [4], Choudhury and Ke [5], and others considered the unreliable queues wherein, when a server fails, customers in the system should wait for the server to be repaired without being served. However, there are other practical situations: when a server breakdown occurs, the system continues to forward some customers without stopping immediately. For example, nonstop forwardingis designed to minimize packet loss during a management system failure by maintaining L2 and L3 forwarding, respectively, where the system continues forwarding some packets in the event of a processor failure $[6,7]$.

Motivated by this factor, we consider a discrete-time N-limited nonstop forwarding, where the service continues instead of being stopped completely even in the case that the server is defective. The server starts immediately the repair process whenever the server breaks down. Despite the server breakdown, up to $\mathbb{N}$ customers can be serviced during the repair time.Lee [8] analyzed a discrete-time N-limited nonstop forwarding queue with batch geometric arrivals and deterministic service times. Lee and Choi [9] analyzed the steady-state distribution of the number of customers in $\mathrm{Geo} / \mathrm{Geo} / 1$ nonstop forwarding queueing system. In this paper, we analyze the delay distribution of customers of Geo/Geo/1 N-limited nonstop forwarding queue.

\section{MODEL}

This paper considers a discrete-time Geo/Geo/1 queueing system in which the time axis is divided into fixed-length contiguous intervals, referred to as slots. Customers arrive according to a geometric process. Let $p$ be the probability that a customer enters the system during a slot. It is assumed that the service of a customer can start only at a slot boundary. The service times of customers are assumed to follow geometric distribution with parameter $q$. The system has a buffer of infinite capacity. Customers are served in FCFS order. The exact location of arrival instants within the slot length are not specified here. It is even irrelevant as long as the system is observed at slot boundaries only.

It is assumed that the server is subject to breakdowns. The server broken down starts immediately the repair process. It is also assumed that the lifetime of the server is geometrically distributed with parameter $\alpha$, where $1-\alpha$ is the probability that a failure does not occur in a slot. The repair times of servers follows a geometrical distribution with parameter $\beta$, where $1-\beta$ is the probability that a failure will not be concluded in a slot. When the server breaks down, the system continues to forward the next $N$ customers. It is also assumed that the inter-arrival times, the service times, the failure times, and the repair times are mutually independent of each other.

Let $M(k)$ be the number of customers in the system at the beginning of slot $k$. Let $S(k)$ be the server state at the beginning of slot $k: S(k)=n$ if the server is under repair and the system has forwarded $\mathrm{N}-\mathrm{n}$ customers after the server's breakdown; $S(k)=N+1$ if the server is normal.Then $\{(M(k), S(k))\}$ is a Markovian process with state space $\{0,1,2\} \times\{0,1, \ldots, N+1\}$. 


\section{DELAY DistRIBUTION}

Under the equilibrium condition $[9,10]$ :

$p<q\left[1-\frac{\alpha}{\alpha+\beta}\left\{\frac{q(1-\beta)}{1-(1-q)(1-\beta)}\right\}^{N}\right]$

we can compute the steady state distribution [9]:

$x_{i, j} \equiv \lim _{k \rightarrow \infty} \mathrm{P}\{M(k)=i, S(k)=j\}$

of the Markov chain.

The delay is defined as the total time period a tagged customer spends in the system, i.e., the number of slots between the end of the customer's arrival slot and the end of its departure slot. Let $d_{i, j}$ be the mean remaining delay of a tagged customer at the beginning of a slot when the number of customers that will be served before the tagged customer is $i-1$ and the system state is $j$ for $i=1,2, \ldots$, and $j=0,1,2, \ldots, N+1$. Letting:

$d_{\mathrm{i}} \equiv\left(d_{\mathrm{i}, 0}, d_{\mathrm{i}, 1}, \ldots, d_{\mathrm{i}, N+1}\right)^{\mathrm{T}}$

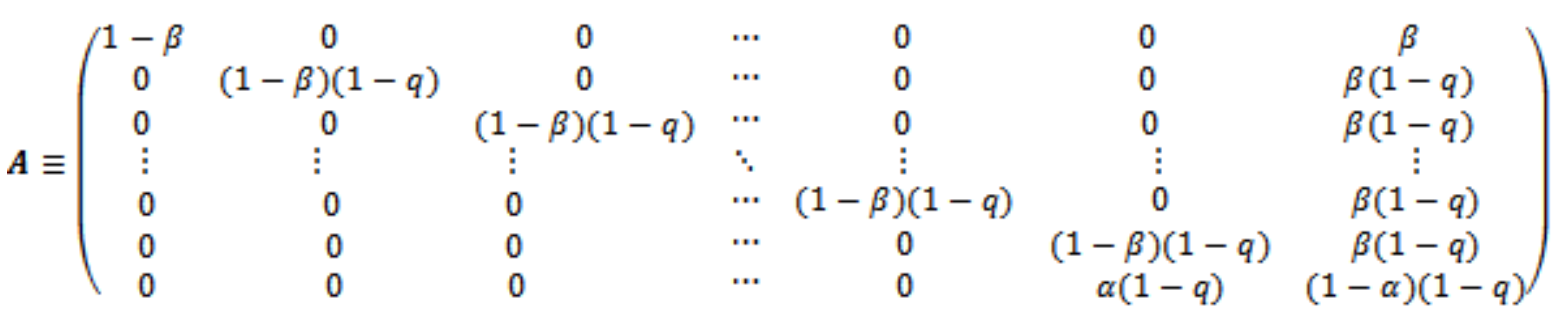

$\boldsymbol{B} \equiv\left(\begin{array}{ccccccc}0 & 0 & 0 & \cdots & 0 & 0 & 0 \\ 1-\beta & 0 & 0 & \cdots & 0 & 0 & \beta \\ 0 & 1-\beta & 0 & \cdots & 0 & 0 & \beta \\ \vdots & \vdots & \vdots & \cdots & \vdots & \vdots & \vdots \\ 0 & 0 & 0 & \cdots & 0 & 0 & \beta \\ 0 & 0 & 0 & \cdots & 1-\beta & 0 & \beta \\ 0 & 0 & 0 & \cdots & 0 & \alpha & 1-\alpha\end{array}\right)$

we obtain:

$d_{1}=A d_{1}+e$

$d_{n}=q B d_{n-1}+A d_{n}+e_{x} \quad n \geq 2$

Hence:

$\boldsymbol{d}_{n}=\sum_{\mathrm{i}=0}^{n-1}\left[(I-A)^{-1} q B\right]^{i}(I-A)^{-1} e_{v} \quad n \geq 1$

Using the stationary probability vector presented above, we now determine the mean delay $\mathrm{E}(D)$. The mean delay $\mathrm{E}(D)$ of a tagged customer is obtained by:

$\mathrm{E}(D)=\sum_{j=0}^{N} x_{0, j}\left\{\beta d_{1, N+1}+(1-\beta) d_{1, j}\right\}+x_{0, N+1}\left\{\alpha d_{1, N}+(1-\alpha) d_{1, N+1}\right\} 1$ 


$$
+\sum_{i=1}^{\infty}\left[\begin{array}{c}
x_{i, 0}\left\{\beta d_{i+1, N+1}+(1-\beta) d_{i+1,0}\right\} \\
+x_{i, N+1}\left\{\alpha q d_{i, N}+(1-\alpha) q d_{i, N+1}+\alpha(1-q) d_{i+1, N}+(1-\alpha)(1-q) d_{i+1, N+1}\right\} \\
+\sum_{j=1}^{N} x_{i, j}\left\{\beta q d_{i, N+1}+(1-\beta) q d_{i, j-1}+\alpha(1-q) d_{i+1, N+1}+(1-\alpha)(1-q) d_{i+1, j}\right\}
\end{array}\right]
$$

IV. Conclusion

Nonstop forwarding is designed to minimize packet loss when a management system fails to function. In the nonstop forwarding mechanism, the system can continue to forward some packets in the event of a processor failure. This paper considered a Geo/Geo/1 N-limited nonstop forwarding queue. This paper gave the delay distribution of customers by matrix geometric analysis.

\section{ACKNOWLEDGEMENT}

This work was supported by the ICT R\&D program of MSIP/IITP. [R0101-15-0070, Development of The High Availability Network Operating System for Supporting Non-Stop Active Routing].

\section{REFERENCES}

[1] C.T. Do, N.H. Tran, C.S. Hong, S. Lee, J.J. Lee, and W. Lee,A lightweight algorithm for probability-based spectrum decision scheme in multiple channels cognitive radio networks, IEEE Commun. Lett., 17(3), 2013, 509-512.

[2] N. Gharbi,B. Nemmouchi,L. Mokdad, and J. Ben-Othman,The impact of breakdowns disciplines and repeated attempts on performance of small cell networks,J. Computational Science,5(4), 2014, 633-644.

[3] G. Falin,An M/G/1 retrial queue with an unreliable server and general repair times,Perform. Evaluation, 67(7), 2010, 569-582.

[4] Y. Lee,Discrete-time bulk-service queue with Markovian service interruption and probabilistic bulk size, $J$. Appl. Math. \& Informatics, 28(1-2), 2010,275-282.

[5] G. Choudhury, and J.C. Ke,An unreliable retrial queue with delaying repair and general retrial times under Bernoulli vacation schedule,Applied Mathematics and Computation, 230, 2014, 436-450.

[6] R. Froom,B. Sivasubramanian, and E. Frahim, Implementing Cisco Switched Networks (SWITCH), CISCO Press, 2010.

[7] R. Surton, K. Birman, and R. van Renesse, Application-driven TCP recovery and non-stop BGP, Proc. 43rd Annual IEEE/IFIP International Conference on Dependable Systems and Networks,2013, 1-12.

[8] Y. Lee, Queueing systems with N-limited nonstop forwarding,East Asian mathematical Journal, 31(5), 2015, 707-716.

[9] Y. Lee and W. Choi, Geo/Geo/1 queue for graceful restart mechanism, Global Journal for Research Analysis, 5(2), 2016, 47-48.

[10] M.F. Neuts,Structured Stochastic Matrices of M/G/1 Type and Their Applications, Marcel Dekker, New York, NY., 1989. 